\title{
BIOLOGICAL NITROGEN FIXATION STABILITY OF COWPEA CULTIVARS WITH TROPICAL SEMI-ARID RHIZOBIAL STRAINS ${ }^{1}$
}

\author{
AMANDA CORDEIRO DE MELO SOUZA ${ }^{2}$, THIAGO PONTES LIRA ${ }^{2}$, ANTONIO FÉLIX DA COSTA ${ }^{3}$, FELIPE JOSÉ \\ CURY FRACETTO ${ }^{2}$, GISELLE GOMES MONTEIRO FRACETTO ${ }^{2}$, MARIO ANDRADE LIRA JUNIOR ${ }^{2 *}$
}

\begin{abstract}
Cowpeas (Vigna unguiculata L. Walp) are an economically and socially important legume in northern and north-eastern Brazil and can establish effective symbiosis with nitrogen-fixing bacteria. We evaluated the symbiotic compatibility and efficiency of rhizobial strains from Pernambuco semi-arid soils and determined their symbiotic stability on the IPA-206, BR 17-Gurguéia, and BRS Novaera cultivars, selected for different environments. The experiment was conducted in a greenhouse to evaluate a $3 \times 28$ factorial arrangement (cultivars selected for different environments $\times$ inoculation with the currently recommended strain, uninoculated plants with or without mineral nitrogen, and 25 rhizobial strains from semi-arid soils) in a randomized block design with four replicates. We determined nodule number, shoot and root dry matter, nodule dry matter by nodule number, nitrogen accumulated in the shoot by nodule dry matter, nitrogen content and accumulation in the shoot, relative efficiency of the recommended strain based on nitrogen accumulation, and shoot dry matter. Overall, the cultivars responded differently to different strains and cultivar biological nitrogen fixation potential. Strains G7.85 and BR 3262 showed potential for biological nitrogen fixation. BR 3262 was confirmed to be adequate for inoculation of different cowpea cultivars.
\end{abstract}

Keywords: Vigna unguiculata. Simbiotic compatibility. IPA-206. BR 17-Gurguéia. BRS Novaera.

\section{ESTABILIDADE DA FIXAÇÃO BIOLÓGICA DE NITROGÊNIO EM CULTIVARES DE FEIJÃO-CAUPI COM LINHAGENS RIZOBIANAS DO SEMIÁRIDO TROPICAL}

RESUMO - O feijão-caupi (Vigna unguiculata L. Walp) é econômica e socialmente importante nas regiões Norte e Nordeste do Brasil e estabelece simbiose com bactérias fixadoras de nitrogênio. Foi avaliada a compatibilidade simbiótica e eficiência de isolados rizobianos de solos do semiárido de Pernambuco e determinada sua estabilidade simbiótica com as cultivares IPA-206, BR 17-Gurguéia e BRS Novaera, selecionadas para diferentes ambientes. $\mathrm{O}$ experimento foi em casa-de-vegetação, avaliando um fatorial $3 \times 28$ (cultivares selecionadas para diferentes ambientes $\times$ inoculação com a estirpe atualmente recomendada, ausência de inoculação com e sem adição de nitrogênio mineral ou inoculação com 25 estirpes de solos do semiárido), em blocos casualizados com quatro repetições. Foram avaliados: número de nódulos, matérias secas da parte aérea, raiz e nódulos, matéria seca de nódulos por número de nódulos, nitrogênio acumulado na parte aérea pela matéria seca de nódulos, teor e acúmulo de nitrogênio na parte aérea, eficiência relativa com base no acúmulo de nitrogênio e eficiência relativa à estirpe recomendada baseada na matéria seca da parte aérea. No geral, as cultivares responderam diferentemente às diferentes linhagens e potencial de fixação biológica de nitrogênio da cultivar. As linhagens G7.85 e BR 3262 apresentaram potencial para fixação biológica de nitrogênio. A confirmação da BR 3262 foi adequada para inoculação do feijão caupi em diferentes cultivares.

Palavras-chave: Vigna unguiculata. Compatibilidade simbiótica. IPA-206. BR 17-Gurguéia. BRS Novaera.

\footnotetext{
*Corresponding author

${ }^{1}$ Received for publication in 11/12/2019; accepted in 01/22/2021.

Paper extracted from the Master's dissertation of the first author.

${ }^{2}$ Department of Agronomy, Universidade Federal Rural de Pernambuco, Recife, PE, Brazil; amanda.cmsouza@yahoo.com.br - ORCID: 0000-0003-0397-097X, thiagopontesliratp1@gmail.com - ORCID: 0000-0001-7330-4222, curyfelipe@ hotmail.com - ORCID: 0000-00029112-1339, giselle.fracetto@ufrpe.br-ORCID: 0000-0001-9366-2588, mario.alirajr@ufrpe.br-ORCID: 0000-0001-7779-3369.

${ }^{3}$ Instituto Agronômico de Pernambuco, Recife, PE, Brazil; afelixc.ipa@gmail.com - ORCID: 0000-0001-9866-3504.
} 


\section{INTRODUCTION}

Cowpeas (Vigna unguiculata (L.) Walp) are an important legume in the cropping systems of tropical semi-arid environments (SAKA et al., 2019). Its annual global production is approximately 7 million tons grown on more than 12 million hectares (FAOSTAT, 2016). It is widely grown in northern and north-eastern Brazil and accounts for $80 \%$ of the cultivated area in Brazil (SILVA et al., 2018). It is very effective in its symbiosis with nitrogen-fixing bacteria, known as rhizobia, is adapted to different soil and climatic conditions (ADU et al., 2019), and has high genetic variability (OMOIGUI et al., 2019).

Legume biological nitrogen fixation (BNF), including in cowpeas, may reduce nitrogen fertilizer usage and reduce greenhouse gas emissions (PUOZAA; JAISWAL; DAKORA, 2019). Cowpeas can nodulate and fix nitrogen with a large bacterial spectrum, but BNF efficiency and efficacy depend on both symbiotic partners being compatible (JI et al., 2019). Thus, inoculation with highly efficient strains might be hampered because widely occurring native strains are usually competitive and, as in any population, have a varying range of BNF potentials (IRISARRI et al., 2019).

The continual screening and evaluation of new rhizobial strains are necessary to select more efficient and adaptable symbiotic associations and sustainably increase grain yield in tropical environments (NASCIMENTO et al., 2019). This is particularly important in the tropics because of the high variability in soils and climate, which leads directly to high biodiversity in the rhizobial population, and thus, the potential to select higher fixing strains for use in inoculant production (NDUNGU et al., 2018).

Cowpea cultivars are recommended for specific planting regions; however, because of national regulations, rhizobial strains for inoculant production are nationally recommended in Brazil (BRASIL, 2011). Hence, there is an unmet need to study the stability of symbioses of different cowpea cultivars $\times$ bacterial strain combinations. Although this analysis is performed more frequently for plant breeding, Lira et al. (1994) evaluated a cultivar by forage cut interaction for forage yield using stability analysis.

We evaluated the symbiotic compatibility, efficiency, and stability of native rhizobial strains isolated from tropical semi-arid soils and a strain currently recommended for Brazilian-wide commercial inoculant production in three cowpea cultivars indicated for widely different cowpea growing regions.

\section{MATERIAL AND METHODS}

Soil samples were collected in 2010 from 11 municipalities in the semi-arid regions of Pernambuco State, Brazil: Santa Cruz, Parnamirim, Serra Talhada, Sertânia, Petrolina, Floresta, Tupanatinga, Jataúba, Santa Cruz do Capibaribe, Bom Jardim, and Caetés. Sampling was performed in each soil class in the municipality, based on the Agroecological Zoning of the State of Pernambuco (SILVA et al., 2001) (Supplementary Material).

Cowpea cultivars IPA-206, BR 17-Gurguéia, and BRS Novaera, indicated for cultivation in Pernambuco, Piauí, and the northern Brazilian region, respectively (GONÇALVES, 2012; IPA, 1989), were inoculated with 25 new rhizobial strains and three control treatments, including inoculation with BR 3262 a Bradyrhizobium sp. strain currently recommended for Brazilian inoculant production (BRASIL, 2011), uninoculated with nitrogen fertilization (CN.SI), or uninoculated without nitrogen fertilization (0N.SI) in a $3 \times 28$ factorial arrangement in a randomized block design with four replicates. The greenhouse conditions were $28{ }^{\circ} \mathrm{C}$ and $68 \%$ relative humidity.

The 25 new isolates were previously selected from a group of 109 isolates from two soil groups, four from high-fertility soils (Floresta, Parnamirim, and Santa Cruz do Capibaribe municipalities) and 21 from low-fertility soils (Caetés, Petrolina, Santa Cruz, Sertânia, and Tupanatinga municipalities). All were from the tropical semi-arid region of Pernambuco State (LIRA, 2014), based on their effects on cowpea cultivar IPA-206, which is recommended for this growth environment under protected environmental conditions. The isolated rhizobia belonged to soils with low or high fertility and were selected because of significant differences in shoot dry matter.

All bacteria were grown in $1 \mathrm{~mL}$ of liquid YM medium (VINCENT, 1970). The $\mathrm{pH}$ was adjusted to 6.8 in $125 \mathrm{~mL}$ Erlenmeyer flasks containing $25 \mathrm{~mL}$ on a rotary shaker at $150 \mathrm{rpm}$ at $30{ }^{\circ} \mathrm{C}$ for $72 \mathrm{~h}$ for later inoculation. Cowpea seeds were disinfected in $70 \%$ ethanol for $1 \mathrm{~min}$, then in $1 \%$ sodium hypochlorite for $2 \mathrm{~min}$ and washed with distilled water. This process was performed to disinfest two seeds per vessel, and subsequently, they were inoculated with $1 \mathrm{~mL}$ of liquid culture medium with known bacterial counts for each seed. Plants were cultivated in a modified Leonard jar with washed sand and vermiculite (2:1 ratio), which were both previously autoclaved $\left(1 \mathrm{~h}, 1 \mathrm{~atm}, 120{ }^{\circ} \mathrm{C}\right)$ supplied with Hoagland's nutrient solution without $\mathrm{N}$ (HOAGLAND; ARNON, 1950), whereas the 
uninoculated, $\mathrm{N}$-fertilized treatment received the equivalent to $90 \mathrm{~kg} \mathrm{ha}^{-1}$ of $\mathrm{N}$ as urea (IBEWIRO et al., 2001) in three equal doses starting seven days after germination with the following two doses applied at $15 \mathrm{~d}$ intervals. For the treatment with fertilization, Hoagland's solution was added. Plants were harvested $45 \mathrm{~d}$ after inoculation, separated into shoots, roots, and nodules, and dried at $65{ }^{\circ} \mathrm{C}$ until they reached a constant dry mass. After weight stabilization, nodule dry matter (NDM), root dry matter (RDM), and shoot dry matter (SDM) were determined. Shoot N content (SNC) was determined using the Kjeldahl method and shoot total N (STN) was calculated by multiplying SDM and SNC.

Derived measures were also calculated: nodule dry matter by nodule number (NDMNN), $\mathrm{N}$ accumulated in the shoots by nodule dry matter (STN:NDM), the relative efficiency of the recommended strain based on nitrogen accumulation (RENA), and relative efficiency of the strain recommended based on shoot dry matter (RESDM).

$$
\begin{aligned}
\text { NDMNN }=\left(\frac{\text { NDM }}{\text { NN }}\right) & \text { STN:NDMN }=\left(\frac{\text { STN }}{\text { NDM }}\right) \\
\text { RESDM }=\left(\frac{\text { SDM inoculated }}{\text { SDM BR 3262 }}\right) & \text { RENA }=\left(\frac{\text { STN inoculated }}{\text { STN BR 3262 }}\right)
\end{aligned}
$$

Data were analyzed using analysis of variance (ANOVA) using the SAS program (SAS Institute, 1999). Means were compared by Dunnett's test at a 5\% significance level, adopting the treatment with the recommended strain as the control for the main effect of strains, whereas the interactions were evaluated using Tukey's test at the same level of significance. For adaptability and stability of the symbiosis analysis, we used the interaction "cultivar $\times$ strains" (EBERHART; RUSSELL, 1966) when there was an interaction between cultivar and strain, considering inoculated treatments and strains as environments for cultivars and the reverse. This methodology was based on a linear regression model.

$$
Y_{i j}=\mu_{i}+\beta_{i} I_{j}+\delta_{i j}
$$

where, Y $i j$ is the mean of genotype "i $i$ " in the environment " $\mathrm{j}$ "; $\mu i$ is the mean of genotype " $\mathrm{i}$ " in all environments; $\beta i$ is the linear regression coefficient of the genotype "i" response to all environments; $\mathrm{I} j$ is the environmental index; $\delta i j$ is the regression deviation of genotype "i" in environment "j."

\section{RESULTS AND DISCUSSION}

Lira (2014) demonstrated that of the 23 isolates selected, G7.25, G7.77, and G7.18 belonging to low-fertility soils showed the best performance in SDM, nodule number, NDM, specific nodule biomass, shoot total nitrogen, and relative efficiency, and were promising for BNF in cowpeas.

Uninoculated treatments did not nodulate (Table 1), whereas the recommended BR 3262 had a high NN, confirming the potential of this strain, as shown in agronomic efficiency tests in several soil and climatic conditions (LEITE et al., 2018). The BR 3262 strain had superior NDM, suggesting high efficiency (CARVALHO et al., 2019), although its NDMNN and STN:NDM were similar to that of the other strains. All cultivars nodulated with all strains, indicating sufficient compatibility independent of strain and cultivar origin for this trait. BNF in cowpea cultivars is recommended for different environmental conditions in Roraima and showed that BR 3262 provided a greater increase in NDM than that of other treatments (MELO; ZILLI, 2009).

Regarding RDM, 15 isolates provided similar dry matter of cowpea roots as did the recommended strain. G7.14, G7.99, and G7.68 isolates obtained equal averages in relation to $\mathrm{BR} 3262$. NN 15 isolates did not differ from BR 3262, with higher mean values for G7.102, G7.109, and G7.32, followed by strain BR 3262 (Table 1). These results corroborate those of Lira (2014), indicating that these isolates are as efficient as the recommended strain. Almeida et al. (2010) found different results for strain BR 3262, with a smaller nodule number in relation to treatments inoculated with BR 17Gurguéia in north-eastern tropical soils.

The plants of all inoculated treatments showed root nodules, which confirmed that all rhizobia studied were compatible with the varieties selected for different environments, despite the rhizobia isolates belonging to the native semi-arid region (Pernambuco State, Brazil). Regarding NDMNN, 20 isolates did not differ from BR 3262, with higher mean values for G7.77 (2.4 mg/nodule) and strain BR $3262(2.1 \mathrm{mg} /$ nodule $)$, followed by isolate G7.68 (2.0 mg/nodule). For STN:NDM, 18 isolates did not differ from the reference strain (Table 1).

Nodule dry mass and nodule number are important variables in evaluating the symbiotic efficiency of rhizobial strains (CAMPO; HUNGRIA, 2006). The recommended strain BR 3262 performed well for the variables, justifying its use in agronomic efficiency tests in several edaphoclimatic conditions (SABOYA et al., 2013). 
Table 1. Root dry matter (RDM), nodule dry matter (NDM), nodule number (NN), nodule dry mass by nodule number (NDMNN), and shoot total $\mathrm{N}$ by nodule dry matter STN: NDM.

\begin{tabular}{|c|c|c|c|c|c|}
\hline Treatments & $\begin{array}{c}\text { RDM } \\
\text { (g plant }^{-1} \text { ) }\end{array}$ & $\begin{array}{c}\text { NDM } \\
\left(\text { mg plant }^{-1}\right)\end{array}$ & $\begin{array}{c}\mathrm{NN} \\
\text { (nodule plant }^{-1} \text { ) }\end{array}$ & $\begin{array}{l}\text { NDMNN } \\
\left(\text { mg plant }^{-1}\right)\end{array}$ & $\begin{array}{l}\text { STN:NDM } \\
\left(\text { mg plant }^{-1}\right)\end{array}$ \\
\hline G7.14 & $0.5 \mathrm{a}$ & $118.6 \mathrm{a}$ & $57.9^{\mathrm{a}}$ & $1.9 \mathrm{a}$ & $0.2 \mathrm{~b}$ \\
\hline G7.118 & $0.4 \mathrm{a}$ & $86.4 \mathrm{a}$ & $45.8^{\mathrm{a}}$ & $1.6 \mathrm{a}$ & $0.3 b$ \\
\hline G7.18 & $0.2 b$ & $5.6 \mathrm{~b}$ & $1.9 \mathrm{~b}$ & $0.7 \mathrm{a}$ & $0.5 b$ \\
\hline G7.79 & $0.2 b$ & $1.0 \mathrm{~b}$ & $0.6 \mathrm{~b}$ & $0.2 b$ & $0.9 \mathrm{a}$ \\
\hline G7.99 & $0.5 \mathrm{a}$ & $91.6 \mathrm{a}$ & $54.5^{\mathrm{a}}$ & $1.7 \mathrm{a}$ & $0.4 b$ \\
\hline G7.12 & $0.4 \mathrm{a}$ & $41.9 \mathrm{a}$ & $25.2^{\mathrm{a}}$ & $1.2 \mathrm{a}$ & $0.4 b$ \\
\hline G7.68 & $0.5 \mathrm{a}$ & $108.7 \mathrm{a}$ & $53.5^{\mathrm{a}}$ & $2.0 \mathrm{a}$ & $0.2 \mathrm{~b}$ \\
\hline G7.45 & $0.2 b$ & $0.9 b$ & $0.4 b$ & $0.2 b$ & $0.8 \mathrm{a}$ \\
\hline G7.103 & $0.2 b$ & $9.6 \mathrm{~b}$ & $5.6 \mathrm{~b}$ & $0.7 \mathrm{a}$ & $0.5 b$ \\
\hline G1.62 & $0.1 \mathrm{~b}$ & $1.4 \mathrm{~b}$ & $1.1 \mathrm{~b}$ & $0.3 b$ & $0.7 \mathrm{a}$ \\
\hline G7.77 & $0.4 \mathrm{a}$ & $142.9 \mathrm{a}$ & $54.5^{\mathrm{a}}$ & $2,4 a$ & $0.3 b$ \\
\hline G7.102 & $0.4 \mathrm{a}$ & $76.7 \mathrm{a}$ & $81.8^{\mathrm{a}}$ & $1.1 \mathrm{a}$ & $0.3 b$ \\
\hline G1.1 & $0.2 b$ & $8.9 b$ & $5.5 \mathrm{~b}$ & $0.5 \mathrm{a}$ & $0.6 \mathrm{a}$ \\
\hline G7.32 & $0.4 \mathrm{a}$ & $145.8 \mathrm{a}$ & $75.0 \mathrm{a}$ & $1.9 \mathrm{a}$ & $0.3 b$ \\
\hline G7.109 & $0.4 \mathrm{a}$ & $119.9 \mathrm{a}$ & $77.9^{\mathrm{a}}$ & $1.5 \mathrm{a}$ & $0.3 b$ \\
\hline G7.64 & $0.3 \mathrm{a}$ & $25.3 \mathrm{a}$ & $14.4^{\mathrm{a}}$ & $1.1 \mathrm{a}$ & $0.4 b$ \\
\hline G7.85 & $0.3 \mathrm{a}$ & $91.6 \mathrm{a}$ & $56.7^{\mathrm{a}}$ & $1.6 \mathrm{a}$ & $0.3 b$ \\
\hline G7.25 & $0.1 b$ & $1.0 \mathrm{~b}$ & $0.6 \mathrm{~b}$ & $0.2 b$ & $0.7 \mathrm{a}$ \\
\hline G7.7 & $0.3 \mathrm{a}$ & $91.7 \mathrm{a}$ & $44.6^{\mathrm{a}}$ & $1.9 \mathrm{a}$ & $0.2 b$ \\
\hline G7.23 & $0.3 \mathrm{a}$ & $58.6 \mathrm{a}$ & $40.5^{\mathrm{a}}$ & $1.2 \mathrm{a}$ & $0.4 b$ \\
\hline G7.13 & $0.3 \mathrm{a}$ & $95.2 \mathrm{a}$ & $55.9^{\mathrm{a}}$ & $1.6 \mathrm{a}$ & $0.3 b$ \\
\hline G1.99 & $0.2 b$ & $9.9 \mathrm{~b}$ & $5.6 \mathrm{~b}$ & $0.7 \mathrm{a}$ & $0.5 b$ \\
\hline G1.2 & $0.4 \mathrm{a}$ & $108.7 \mathrm{a}$ & $62.5^{\mathrm{a}}$ & $1.6 \mathrm{a}$ & $0.2 b$ \\
\hline G7.69 & $0.1 b$ & $2.1 \mathrm{~b}$ & $2.2 b$ & $0.2 \mathrm{a}$ & $0.7 \mathrm{a}$ \\
\hline G7.3 & $0.2 b$ & $2.9 b$ & $1.6 \mathrm{~b}$ & $0.4 b$ & $0.7 \mathrm{a}$ \\
\hline BR3262 & $0.5 \mathrm{a}$ & $146.4 \mathrm{a}$ & $64.2^{\mathrm{a}}$ & $2.1 \mathrm{a}$ & $0.2 b$ \\
\hline 0N.SI & $0.1 b$ & $0 \mathrm{~b}$ & $0 \mathrm{~b}$ & $0 \mathrm{~b}$ & - \\
\hline CN.SI & $0.2 b$ & $0 \mathrm{~b}$ & $0 \mathrm{~b}$ & $0 \mathrm{~b}$ & - \\
\hline VC $(\%)$ & 16.5 & 49.3 & 45.2 & 36.1 & 26.3 \\
\hline
\end{tabular}

Means followed by the same lowercase letter did not differ significantly $(P<0.05)$ by Dunnett's test; VC $=$ variance coefficient.

Although nodulation was not subject to an interaction between cultivar and isolate, SDM was, with different cultivars achieving better results with different rhizobial isolates. The highest SDM occurred for G7.85, IPA-206, and BR 17-Gurguéia, whereas BRS Novaera BR 3262 had a significantly higher SDM (Table 2), confirming the high potential of the latter strain (FERREIRA et al., 2019).

Regarding SDM, the BR 17-Gurguéia variety presented 17 treatments that did not differ from the BR 3262 treatment. For IPA-206, 16 treatments did not differ from the recommended treatment. For BRS Novaera, 15 treatments did not differ from BR 3262 (Table 2). Different results were obtained in field experiments in Maranhão and Roraima States, Brazil, where BR 3262 had greater SDM (GUALTER et al., 2011).

Strains G7.118, G7.18, G7.99, G1.1, G1.99, G1.2, G7.69, and G7.3 had higher SNC values than BR 3262 in both IPA-206 and BRS Novaera, with IPA-206 higher than BRS Novaera, except for G7.3 (Table 3). For STN, G1.2 provided better performance in BRS Novaera, and the BR 3262 strain exhibited better performance in BR 17Gurguéia, and G7.14, G7.118, G7.99, G7.12, G7.68, G7.77, G7.109, G7.85, G7.23, and G7.13 provided better performance in IPA-206 (Table 3).

For SNC, the variety BR 17-Gurguéia presented 25 treatments that did not differ from BR 3262. Variety IPA-206 had 23 treatments that did not differ from the strain BR 3262, and variety BRS Novaera had 25 treatments inoculated with isolates that did not differ from treatment with BR 3262 (Table 3). All inoculated treatments, except G7.45 with the IPA-206 variety, presented levels above the values indicated as suitable for the culture, being between $1.8 \%$ and 2.2\% (MALAVOLTA et al., 1997), indicating that the BNF was sufficient. For STN, variety BR 17-Gurguéia and reference strain BR 3262 had the highest absolute value; however, 17 treatments did not differ from BR 3262. For variety IPA-206, 15 treatments did not differ from BR 3262. In the BRS Novaera variety, 23 inoculated treatments did not differ from BR 3262 (Table 3). 
The RENA and RESDM values obtained confirmed that BNF met the $\mathrm{N}$ demand of the plants (Table 4); thus, most strains were efficient (CHAGAS JUNIOR et al., 2010), and several did not differ from BR 3262. This indicated a positive response of the cultivars to native semi-arid soil rhizobia strains, regardless of the area to which the cultivar was most adapted.

Table 2. Shoot dry matter (SDM) of BR 17-Gurguéia, IPA-206, and BRS Novaera cowpea cultivars with different rhizobial strains.

\begin{tabular}{cccc}
\hline Treatments & BR 17-Gurguéia & IPA-206 & BRS Novaera \\
\hline G7.14 & $4.34 \mathrm{aA}$ & $4.64 \mathrm{aA}$ & $4.59 \mathrm{aA}$ \\
G7.118 & $3.96 \mathrm{aA}$ & $5.35 \mathrm{aA}$ & $3.44 \mathrm{abA}$ \\
G7.18 & $0.79 \mathrm{cdeA}$ & $0.22 \mathrm{eA}$ & $0.89 \mathrm{cdA}$ \\
G7.79 & $0.48 \mathrm{eA}$ & $0.54 \mathrm{deA}$ & $0.73 \mathrm{cdA}$ \\
G7.99 & $3.79 \mathrm{abA}$ & $3.89 \mathrm{abA}$ & $4.28 \mathrm{abA}$ \\
G7.12 & $3.48 \mathrm{abcA}$ & $3.15 \mathrm{abcA}$ & $1.97 \mathrm{abcdA}$ \\
G7.68 & $3.38 \mathrm{abcA}$ & $4.03 \mathrm{aA}$ & $4.25 \mathrm{abA}$ \\
G7.45 & $0.27 \mathrm{eA}$ & $0.42 \mathrm{eA}$ & $0.70 \mathrm{dA}$ \\
G7.103 & $2.05 \mathrm{abcdeA}$ & $0.79 \mathrm{cdeA}$ & $1.89 \mathrm{abcdA}$ \\
G1.62 & $0.45 \mathrm{eA}$ & $0.38 \mathrm{eA}$ & $1.20 \mathrm{bcdA}$ \\
G7.77 & $4.01 \mathrm{aA}$ & $4.03 \mathrm{aA}$ & $3.90 \mathrm{abA}$ \\
G7.102 & $3.83 \mathrm{abA}$ & $4.11 \mathrm{aA}$ & $4.38 \mathrm{abA}$ \\
G1.1 & $1.67 \mathrm{abcdeA}$ & $0.96 \mathrm{bcdeA}$ & $0.73 \mathrm{cdA}$ \\
G7.32 & $4.17 \mathrm{aA}$ & $2.90 \mathrm{abcA}$ & $4.13 \mathrm{abA}$ \\
G7.109 & $4.69 \mathrm{aA}$ & $3.38 \mathrm{abcA}$ \\
G7.64 & $4.06 \mathrm{aA}$ & $2.75 \mathrm{abcdA}$ & $1.02 \mathrm{bcdA}$ \\
G7.85 & $3.09 \mathrm{abcdA}$ & $3.67 \mathrm{abA}$ & $2.14 \mathrm{abcdA}$ \\
G7.25 & $4.25 \mathrm{aA}$ & $0.43 \mathrm{eA}$ & $0.65 \mathrm{dA}$ \\
G7.7 & $0.28 \mathrm{eA}$ & $3.68 \mathrm{abA}$ & $3.31 \mathrm{abcA}$ \\
G7.23 & $3.20 \mathrm{abcdA}$ & $3.83 \mathrm{abA}$ & $3.29 \mathrm{abcA}$ \\
G7.13 & $3.85 \mathrm{abA}$ & $3.66 \mathrm{abA}$ & $2.71 \mathrm{abcdA}$ \\
G1.99 & $3.27 \mathrm{abcdA}$ & $2.50 \mathrm{abcdA}$ & $1.06 \mathrm{bcdA}$ \\
G1.2 & $0.72 \mathrm{deA}$ & $4.30 \mathrm{aA}$ & $4.24 \mathrm{abA}$ \\
G7.69 & $3.47 \mathrm{abcA}$ & $0.57 \mathrm{deA}$ & $0.90 \mathrm{cdA}$ \\
G7.3 & $0.83 \mathrm{bcdeA}$ & $0.76 \mathrm{cdeA}$ & $0.88 \mathrm{cdA}$ \\
BR3262 & $0.40 \mathrm{eA}$ & $3.65 \mathrm{abA}$ & $4.62 \mathrm{aA}$ \\
0 N.SI & $0.30 \mathrm{eA}$ & $1.02 \mathrm{bcdA}$ \\
CN.SI & $4.21 \mathrm{aA}$ & $0.65 \mathrm{cdeA}$ & $1.60 \mathrm{abcdA}$ \\
\hline
\end{tabular}

Means followed by the same lowercase letter in the columns and uppercase letters in the rows, do not differ according to Tukey's test at a $5 \%$ probability.

Table 3. Shoot nitrogen content (SNC) and shoot total nitrogen (STN) of BR 17-Gurguéia, IPA-206, and BRS Novaera cowpea cultivars with different rhizobial strains.

\begin{tabular}{|c|c|c|c|c|c|c|}
\hline \multirow{2}{*}{ Treatments } & \multicolumn{3}{|c|}{ SNC (\%) } & \multicolumn{3}{|c|}{ STN (mg/plant) } \\
\hline & BR 17-Gurguéia & IPA-206 & BRS Novaera & BR 17-Gurguéia & IPA-206 & $\overline{\text { BRS Novaera }}$ \\
\hline G7.14 & $3.66 \mathrm{abcA}$ & $3.09 \mathrm{abA}$ & $3.02 \mathrm{abA}$ & $107.56 \mathrm{aA}$ & $99.89 \mathrm{bA}$ & $97.85 \mathrm{abA}$ \\
\hline G7.118 & $3.91 \mathrm{abA}$ & $3.55 \mathrm{abA}$ & $4.10 \mathrm{aA}$ & $105.54 \mathrm{aA}$ & $83.45 \mathrm{bcA}$ & $100.93 \mathrm{abA}$ \\
\hline G7.18 & $2.57 \mathrm{abcA}$ & 3.09abA & $4.31 \mathrm{aA}$ & $14.40 \mathrm{bcdA}$ & $11.67 \mathrm{def} A$ & $26.03 \mathrm{abcA}$ \\
\hline G7.79 & $2.65 \mathrm{abcA}$ & $2.23 \mathrm{bA}$ & $3.71 \mathrm{abA}$ & $9.38 \mathrm{dA}$ & $8.12 \mathrm{efA}$ & $18.83 \mathrm{bcA}$ \\
\hline G7.99 & $3.25 \mathrm{abcA}$ & $3.60 \mathrm{abA}$ & $3.65 \mathrm{abA}$ & $85.68 \mathrm{abA}$ & $97.99 \mathrm{bA}$ & 71.33abcA \\
\hline G7.12 & $3.88 \mathrm{abA}$ & $3.60 \mathrm{abA}$ & $3.40 \mathrm{abA}$ & $95.61 \mathrm{abA}$ & $79.41 \mathrm{bcdA}$ & 44.88abcA \\
\hline G7.68 & $4.12 \mathrm{aA}$ & $3.48 \mathrm{abA}$ & $3.11 \mathrm{abA}$ & $96.14 \mathrm{aA}$ & $98.36 \mathrm{bA}$ & $90.07 \mathrm{abcA}$ \\
\hline G7.45 & $3.01 \mathrm{abcA}$ & $1.71 \mathrm{bA}$ & $3.70 \mathrm{abA}$ & $5.72 \mathrm{dA}$ & $6.96 \mathrm{fA}$ & $17.78 \mathrm{bcA}$ \\
\hline G7.103 & 3.64abcA & $3.00 \mathrm{abA}$ & $3.47 \mathrm{abA}$ & 52.18abcdA & $16.33 \mathrm{cdef} A$ & 46.13abcA \\
\hline G1.62 & $3.07 \mathrm{abcA}$ & $2.85 \mathrm{abA}$ & $4.24 \mathrm{aA}$ & $9.70 \mathrm{cdA}$ & 7.59efA & 34.66abcA \\
\hline G7.77 & $2.82 \mathrm{abcA}$ & $3.60 \mathrm{abA}$ & 3.39abA & $78.62 \mathrm{abcA}$ & $101.39 \mathrm{abA}$ & 91.84abcA \\
\hline G7.102 & $4.16 \mathrm{aA}$ & $3.10 \mathrm{abA}$ & $3.86 \mathrm{aA}$ & $109.81 \mathrm{aA}$ & $88.46 \mathrm{bA}$ & $117.96 \mathrm{aA}$ \\
\hline
\end{tabular}

Means followed by the same lowercase letter in the columns and uppercase letters in the rows, do not differ according to Tukey's test at a $5 \%$ probability. 
Table 3. Continuation.

\begin{tabular}{|c|c|c|c|c|c|c|}
\hline \multirow{2}{*}{ Treatments } & \multicolumn{3}{|c|}{ SNC (\%) } & \multicolumn{3}{|c|}{ STN (mg/plant) } \\
\hline & BR 17-Gurguéia & IPA-206 & BRS Novaera & BR 17-Gurguéia & IPA-206 & BRS Novaera \\
\hline G1.1 & $3.73 \mathrm{abA}$ & $3.60 \mathrm{abA}$ & $4.18 \mathrm{aA}$ & 43.78abcdA & $23.58 \mathrm{cdefA}$ & 19.84abcA \\
\hline G7.32 & $3.98 \mathrm{aA}$ & $3.26 \mathrm{abA}$ & $3.83 \mathrm{aA}$ & $114.64 \mathrm{aA}$ & $64.00 \mathrm{bcdefA}$ & $109.64 \mathrm{aA}$ \\
\hline G7.109 & $4.11 \mathrm{aA}$ & $2.87 \mathrm{abA}$ & $3.03 \mathrm{abA}$ & $114.60 \mathrm{aA}$ & $92.90 \mathrm{bA}$ & 73.92abcA \\
\hline G7.64 & $4.10 \mathrm{aA}$ & $2.56 \mathrm{abA}$ & $3.29 \mathrm{abA}$ & $87.41 \mathrm{abA}$ & $45.08 \mathrm{cdefA}$ & 23.34abcA \\
\hline G7.85 & $4.04 \mathrm{aA}$ & $3.43 \mathrm{abA}$ & $3.17 \mathrm{abA}$ & $118.35 \mathrm{aA}$ & $87.26 \mathrm{bcA}$ & 48.26abcA \\
\hline G7.25 & $3.73 \mathrm{abA}$ & $3.43 \mathrm{abA}$ & $3.32 \mathrm{abA}$ & $7.36 \mathrm{dA}$ & $10.50 \mathrm{efA}$ & $15.04 \mathrm{cA}$ \\
\hline G7.7 & $4.14 \mathrm{aA}$ & $2.76 \mathrm{abA}$ & $3.61 \mathrm{abA}$ & $91.05 \mathrm{abA}$ & $72.85 \mathrm{bcdeA}$ & $83.12 \mathrm{abcA}$ \\
\hline G7.23 & $3.98 \mathrm{aA}$ & $2.95 \mathrm{abA}$ & $3.82 \mathrm{aA}$ & 69.70abcdA & 79.98bcdA & 87.35abcA \\
\hline G7.13 & $3.76 \mathrm{abA}$ & $3.47 \mathrm{abA}$ & $3.12 \mathrm{abA}$ & 85.62abcA & $88.6 \mathrm{bA}$ & 59.66abcA \\
\hline G1.99 & $2.88 \mathrm{abcA}$ & $3.66 \mathrm{abA}$ & $3.70 \mathrm{abA}$ & $14.62 \mathrm{bcdA}$ & $63.78 \mathrm{bcdefA}$ & 26.03abcA \\
\hline G1.2 & $4.26 \mathrm{aA}$ & $3.88 \mathrm{abA}$ & $4.00 \mathrm{aA}$ & $100.41 \mathrm{aA}$ & $114.41 \mathrm{aA}$ & $117.19 \mathrm{aA}$ \\
\hline G7.69 & $2.58 \mathrm{abcA}$ & $4.91 \mathrm{aA}$ & $3.51 \mathrm{abA}$ & $15.51 \mathrm{bcdA}$ & $14.65 \mathrm{cdefA}$ & 21.62abcA \\
\hline G7.3 & $3.18 \mathrm{abcA}$ & $3.60 \mathrm{abA}$ & $4.02 \mathrm{aA}$ & $8.87 \mathrm{dA}$ & $18.66 \mathrm{bA}$ & $24.51 \mathrm{abcA}$ \\
\hline BR3262 & $4.15 \mathrm{aA}$ & $3.06 \mathrm{abA}$ & $3.41 \mathrm{abA}$ & $118.59 \mathrm{aA}$ & 77.89bcdA & $111.40 \mathrm{aA}$ \\
\hline 0N.SI & $1.19 b c$ & $1.45 \mathrm{bA}$ & $1.37 \mathrm{bA}$ & $2.18 \mathrm{dA}$ & $2.86 \mathrm{fA}$ & $10.50 \mathrm{cA}$ \\
\hline CN.SI & $1.59 \mathrm{cA}$ & $1.83 \mathrm{bA}$ & $2.97 \mathrm{abA}$ & $4.32 \mathrm{dA}$ & $8.90 \mathrm{efA}$ & $33.57 \mathrm{abcA}$ \\
\hline
\end{tabular}

Means followed by the same lowercase letter in the columns and uppercase letters in the rows, do not differ according to Tukey's test at a 5\% probability.

Table 4. Relative efficiency based on nitrogen accumulation (RENA) and relative efficiency of the recommended strain based on shoot dry matter (RESDM) of BR 17-Gurguéia, IPA-206, and BRS Novaera cowpea cultivars with different rhizobial strains.

\begin{tabular}{|c|c|c|c|c|c|c|}
\hline \multirow{2}{*}{ Treatments } & \multicolumn{3}{|c|}{ RENA (\%) } & \multicolumn{3}{|c|}{ RESDM (\%) } \\
\hline & BR 17-Gurguéia & IPA-206 & BRS Novaera & BR 17-Gurguéia & IPA-206 & BRS Novaera \\
\hline G7.14 & $55.81 \mathrm{aA}$ & $80.19 \mathrm{aA}$ & $51.15 \mathrm{abA}$ & $100.80 \mathrm{aA}$ & $128.68 \mathrm{aA}$ & $93.48 \mathrm{aA}$ \\
\hline G7.118 & $54.76 \mathrm{aA}$ & 66.99abcA & $52.88 \mathrm{abA}$ & $91.00 \mathrm{aA}$ & $149.65 \mathrm{aA}$ & 42.13abcA \\
\hline G7.18 & $7.47 \mathrm{cdef} A$ & $9.36 \mathrm{cA}$ & $13.61 \mathrm{abcA}$ & $17.08 \mathrm{bcdA}$ & $4.44 \mathrm{eA}$ & $16.61 \mathrm{bcA}$ \\
\hline G7.79 & $4.87 \mathrm{efA}$ & $6.52 \mathrm{cA}$ & $9.84 \mathrm{abcA}$ & $9.17 \mathrm{cdA}$ & $11.75 \mathrm{deA}$ & $12.12 \mathrm{cA}$ \\
\hline G7.99 & 44.46abcdA & $78.66 \mathrm{aA}$ & 37.29abcA & $87.22 \mathrm{abA}$ & $105.91 \mathrm{abA}$ & $57.86 \mathrm{abcA}$ \\
\hline G7.12 & $49.72 \mathrm{abA}$ & $63.75 \mathrm{abcA}$ & $23.46 \mathrm{abcA}$ & $78.55 \mathrm{abA}$ & 86.06abcdA & $40.00 \mathrm{abcA}$ \\
\hline G7.68 & $49.88 \mathrm{abA}$ & $78.96 \mathrm{aA}$ & 47.09abA & 76.74abA & $109.75 \mathrm{aA}$ & 86.03abA \\
\hline G7.45 & $2.97 \mathrm{fA}$ & $5.59 \mathrm{cA}$ & $9.30 \mathrm{abcA}$ & $4.62 \mathrm{dA}$ & $8.91 \mathrm{eA}$ & $11.63 \mathrm{cA}$ \\
\hline G7.103 & 27.08abcdeA & $13.11 \mathrm{cA}$ & $24.12 \mathrm{abcA}$ & 44.68abcdA & $20.77 \mathrm{cdeA}$ & 37.95abcA \\
\hline G1.62 & 5.03 dcefA & $6.10 \mathrm{cA}$ & $18.12 \mathrm{abcA}$ & $8.25 \mathrm{cdA}$ & $8.20 \mathrm{eA}$ & $21.27 \mathrm{abcA}$ \\
\hline G7.77 & 40.79abcde & $81.39 \mathrm{aA}$ & $48.01 \mathrm{abA}$ & $92.59 \mathrm{aA}$ & $110.13 \mathrm{aA}$ & $80.38 \mathrm{abA}$ \\
\hline G7.102 & $56.98 \mathrm{aA}$ & 71.02abA & $61.61 \mathrm{aA}$ & $88.15 \mathrm{abA}$ & $112.66 \mathrm{aA}$ & $88.76 \mathrm{abA}$ \\
\hline G1.1 & 22.71abcdeA & 18.93abcA & $10.37 \mathrm{abcA}$ & 35.44abcdA & $24.35 \mathrm{bcdeA}$ & $12.30 \mathrm{bcA}$ \\
\hline G7.32 & $59.48 \mathrm{aA}$ & $51.38 \mathrm{abcA}$ & $57.32 \mathrm{abA}$ & $96.69 \mathrm{aA}$ & 77.12abcdA & 83.77abA \\
\hline G7.109 & $59.46 \mathrm{aA}$ & $74.58 \mathrm{abA}$ & $38.64 \mathrm{abcA}$ & $93.53 \mathrm{aA}$ & $130.16 \mathrm{aA}$ & $68.47 \mathrm{abcA}$ \\
\hline G7.64 & $45.35 \mathrm{abcA}$ & 36.19abcA & $12.20 \mathrm{abcA}$ & 69.00abcA & 75.89abcdA & $17.76 \mathrm{bcA}$ \\
\hline G7.85 & $61.41 \mathrm{aA}$ & $70.05 \mathrm{abA}$ & $25.23 \mathrm{abcA}$ & $99.39 \mathrm{aA}$ & $99.85 \mathrm{abA}$ & $41.11 \mathrm{abcA}$ \\
\hline G7.25 & $3.82 \mathrm{efA}$ & $8,3 \mathrm{cA}$ & $7.86 \mathrm{bcA}$ & $5.00 \mathrm{dA}$ & $9.44 \mathrm{eA}$ & $10.63 \mathrm{cA}$ \\
\hline G7.7 & $47.24 \mathrm{abcA}$ & $58.48 \mathrm{abcA}$ & $43.45 \mathrm{abA}$ & $72.80 \mathrm{abA}$ & $100.89 \mathrm{abA}$ & $65.03 \mathrm{abcA}$ \\
\hline G7.23 & 36.17abcdeA & 64.20abcA & $45.66 \mathrm{abA}$ & $60.40 \mathrm{abcA}$ & $103.51 \mathrm{abA}$ & $64.47 \mathrm{abcA}$ \\
\hline G7.13 & 44.43abcdA & $71.25 \mathrm{abA}$ & 31.19abcA & 74.32abA & 98.74abcA & 53.32abcA \\
\hline G1.99 & 7.59cdefA & $52.10 \mathrm{abcA}$ & $13.61 \mathrm{abcA}$ & $14.69 \mathrm{bcdA}$ & 67.95abcdeA & $20.14 \mathrm{abcA}$ \\
\hline G1.2 & $52.10 \mathrm{aA}$ & $91.84 \mathrm{aA}$ & 61.26abA & 78.49abA & $118.44 \mathrm{aA}$ & 86.34abA \\
\hline G7.69 & $8.05 \mathrm{bcdefa}$ & $11.76 \mathrm{cA}$ & $11.30 \mathrm{abcA}$ & $16.64 \mathrm{bcdA}$ & $12.90 \mathrm{deA}$ & $15.82 \mathrm{bcA}$ \\
\hline G7.3 & $4.60 \mathrm{efA}$ & $14.98 \mathrm{bcA}$ & $12.81 \mathrm{abcA}$ & $7.07 \mathrm{cdA}$ & $17.72 \mathrm{deA}$ & $15.41 \mathrm{bcA}$ \\
\hline BR3262 & $61.53 \mathrm{aA}$ & $62.53 \mathrm{abcA}$ & $58.24 \mathrm{abA}$ & $97.99 \mathrm{aA}$ & $98.77 \mathrm{abcA}$ & $95.36 \mathrm{aA}$ \\
\hline 0N.SI & $1.13 \mathrm{fA}$ & $2.30 \mathrm{cA}$ & $5.49 \mathrm{cA}$ & $4.28 \mathrm{dA}$ & $6.25 \mathrm{eA}$ & $18.18 \mathrm{bcA}$ \\
\hline CN.SI & $2.24 \mathrm{fA}$ & $7.4 \mathrm{cA}$ & $17.55 \mathrm{abcA}$ & $6.78 \mathrm{cdA}$ & $14.57 \mathrm{deA}$ & $29.38 \mathrm{abcA}$ \\
\hline
\end{tabular}

Means followed by the same lowercase letter in the columns and uppercase letters in the rows, do not differ according to Tukey's test at a $5 \%$ probability. 
Some studies have shown that plants inoculated with efficient strains, followed by nitrogen treatment and without inoculation, were superior to plants without inoculation and originated BNF (SANTOS et al., 2005; SOARES et al., 2006). Here, nitrogen-supplied plants were among the lowest for SNC and RENA, which is consistent with the results obtained by Sena et al. (2020), who worked with Bradyrhizobium strains in symbiosis with cowpeas. They found that uninoculated and unfertilized plants had the lowest values, as expected for the system.

Most of the isolates showed great efficiency in nitrogen fixation, similar to that observed for strain BR 3262, indicating the existence of efficient rhizobia in cowpea plants. These results confirm the positive response of the varieties to the recommended strain and the isolates, indicating efficiency in the assimilation of atmospheric nitrogen. The isolates that showed low efficiency were less efficient (CHAGAS JUNIOR et al., 2010).

IPA-206 had a higher SDM and SNC in different inoculation treatments; however, for SDM, only BRS Novaera exhibited a regression coefficient $\left(\Omega_{1 \mathrm{i}}\right)$ greater than one $\left(\Omega_{1 \mathrm{i}}>1\right)$, indicating adaptability to higher efficiency strains regardless of different isolation environments, whereas BR 17-Gurguéia showed stability for $\operatorname{SDM}\left(\sigma_{\mathrm{di}}^{2}=0\right)$ and was close to stability for SNC (Table 5). For STN, all cultivars had means equal to zero and were adapted to diverse strains serving as environments (Table 5). In contrast, only IPA-206 had a regression coefficient $\left(\beta_{1 \mathrm{i}}\right)$ greater than one $\left(\beta_{1 \mathrm{i}}>1\right)$ and higher means, indicating adaptability, with low stability for RENA (Table 5), and thus, a greater response to more efficient strains for this characteristic.

Some studies have observed great variability in SNC and STN responses in different varieties of cowpeas associated with selected strains. Because of the relationship between these factors and BFN efficiency, identifying promising symbiotic associations is recommended (STAMFORD; NEPTUNE, 1979; VIEIRA et al., 2010).

Table 5. Adaptability and stability estimates of shoot dry matter (SDM), shoot nitrogen content (SNC), and shoot total nitrogen (STN), relative efficiency to the recommended based on the nitrogen accumulation (RENA), and relative efficiency to the strain recommended based on shoot dry matter (RESDM) of BR 17-Gurguéia, IPA-206, and BRS Novaera cowpea cultivars, according to Eberhart e Russel (1966), considering inoculation as the environments.

\begin{tabular}{|c|c|c|c|}
\hline \multirow{2}{*}{ Cultivars } & \multirow{2}{*}{ Mean } & \multicolumn{2}{|c|}{ SDM } \\
\hline & & $\beta_{1 \mathrm{i}}$ & $\sigma_{\mathrm{di}}^{2}$ \\
\hline BR 17-Gurguéia & 2.37 & $-0.94 *$ & $0.00^{*}$ \\
\hline IPA-206 & 7.63 & $-0.72 *$ & $0.50^{\mathrm{ns}}$ \\
\hline BRS Novaera & 6.29 & $0.34 *$ & $0.71^{\mathrm{ns}}$ \\
\hline General mean & 3.10 & & \\
\hline \multirow{2}{*}{ Cultivars } & \multirow{2}{*}{ Mean } & \multicolumn{2}{|c|}{ SNC } \\
\hline & & $\beta_{1 \mathrm{i}}$ & $\sigma_{\mathrm{di}}^{2}$ \\
\hline BR 17-Gurguéia & 2.22 & $-0.99 *$ & $0.02^{*}$ \\
\hline IPA-206 & 7.64 & $-1.00 *$ & $0.22 *$ \\
\hline BRS Novaera & 6.89 & $-0.88 *$ & $0.21 *$ \\
\hline General mean & 3.48 & & \\
\hline \multirow{2}{*}{ Cultivars } & \multirow{2}{*}{ Mean } & \multicolumn{2}{|c|}{ STN } \\
\hline & & $\beta_{1 \mathrm{i}}$ & $\sigma_{\mathrm{di}}^{2}$ \\
\hline BR 17-Gurguéia & 109.22 & $0.00 *$ & $0.00^{*}$ \\
\hline IPA-206 & 109.22 & $0.00 *$ & $0.00 *$ \\
\hline BRS Novaera & 109.22 & $0.00 *$ & $0.00 *$ \\
\hline General mean & 109.22 & & \\
\hline \multirow{2}{*}{ Cultivars } & \multirow{2}{*}{ Mean } & \multicolumn{2}{|c|}{ RENA } \\
\hline & & $\beta_{1 \mathrm{i}}$ & $\sigma_{\mathrm{di}}^{2}$ \\
\hline BR 17-Gurguéia & 51.26 & $-0.37 *$ & $0.11^{\mathrm{ns}}$ \\
\hline IPA-206 & 86.31 & $0.36^{\mathrm{ns}}$ & $2.73^{*}$ \\
\hline BRS Novaera & 54.91 & $-11.64 *$ & $4.73 *$ \\
\hline General mean & 63.44 & & \\
\hline \multirow{2}{*}{ Cultivars } & \multirow{2}{*}{ Mean } & \multicolumn{2}{|c|}{ RESDM } \\
\hline & & $\beta_{1 \mathrm{i}}$ & $\sigma_{\mathrm{di}}^{2}$ \\
\hline BR 17-Gurguéia & 54.48 & $0.05^{*}$ & $0.19^{\mathrm{ns}}$ \\
\hline IPA-206 & 79.51 & $0.64 *$ & $3.01^{\mathrm{ns}}$ \\
\hline BRS Novaera & 53.87 & $-10.32^{\mathrm{ns}}$ & $4.88^{*}$ \\
\hline General mean & 61.86 & & \\
\hline
\end{tabular}

ns: not significant. * Significant at $5 \%$ probability by $t$-test for $\beta_{1 \mathrm{i}}$ (adaptability) and $\mathrm{F}$ test for $\sigma_{\mathrm{di}}^{2}$ (stability).

Rev. Caatinga, Mossoró, v. 34, n. 2, p. 359 - 369, abr. - jun., 2021 
When the cultivars were considered environments for the strains, 20 strains were considered adapted to below-average environments, considered to be lower BNF efficiency cultivars, and all were close to stability for SDM and SNC (Table 6). Regarding STN, strains presented adaptability to adverse environments, except for G7.12, G7.68, G7.102, G7.32, G7.85, G7.7, G7.13, G7.23, and strain BR 3262, all having means above 109.22 (Table 6), indicating these strains should have even better responses in cultivars with higher BNF potential.

Thus, the greater the potential for BFN in the cultivar, the more the strain will respond, and consequently, the performance of these strains improves in response to cultivars with greater potential. All isolates showed significant regression deviations $\left(\sigma^{2} \mathrm{di} \neq 0\right)$, confirming low stability in response to different cultivars (Table 6). Thus, the isolates will respond positively to varieties with a greater potential for BNF.

Table 6. Adaptability and stability estimates of shoot dry matter (SDM), shoot nitrogen content (SNC), and shoot total nitrogen (STN), relative efficiency to the recommended based on the nitrogen accumulation (RENA), and relative efficiency to the strain recommended based on shoot dry matter (RESDM) of rhizobial strains, according to Eberhart e Russel (1966), considering cultivars as environments.

\begin{tabular}{|c|c|c|c|c|c|c|c|c|c|}
\hline \multirow{2}{*}{ Strains } & \multicolumn{3}{|c|}{ SDM } & \multicolumn{3}{|c|}{$\mathrm{SNC}$} & \multicolumn{3}{|c|}{ STN } \\
\hline & Mean & $\beta_{1 \mathrm{i}}$ & $\sigma_{\mathrm{di}}^{2}$ & Mean & $\beta_{1 \mathrm{i}}$ & $\sigma_{\mathrm{di}}^{2}$ & Mean & $\beta_{1 \mathrm{i}}$ & $\sigma_{\mathrm{di}}^{2}$ \\
\hline G7.14 & 5.88 & $-1.04 *$ & $0.07^{*}$ & 3.92 & $-0.93 *$ & $0.04 *$ & 168.34 & $-0.77 *$ & $0.63^{\mathrm{ns}}$ \\
\hline G7.118 & 4.80 & $-1.06^{*}$ & $0.08 *$ & 4.46 & $-0.95^{*}$ & $0.04 *$ & 167.39 & $-1.64 *$ & $1.16^{\mathrm{ns}}$ \\
\hline G7.18 & 1.40 & $-0.92 *$ & $0.06^{*}$ & 3.98 & $-1.10 *$ & $0.05 *$ & 43.11 & $-0.57 *$ & $0.57^{\mathrm{ns}}$ \\
\hline G7.79 & 1.44 & $-0.99 *$ & $0.01 *$ & 3.55 & $-0.99 *$ & $0.06^{*}$ & 26.60 & $-0.82 *$ & $0.13 *$ \\
\hline G7.99 & 4.92 & $-1.03 *$ & $0.06^{*}$ & 4.14 & $-1.00 *$ & $0.04 *$ & 150.11 & $-1.65^{*}$ & $1.50^{\mathrm{ns}}$ \\
\hline G7.12 & 4.96 & $-0.98 *$ & $0.14^{*}$ & 4.25 & $-0.97 *$ & $0.03 *$ & 129.84 & $2.01 *$ & $3.05^{\mathrm{ns}}$ \\
\hline G7.68 & 5.13 & $-1.11 *$ & $0.06^{*}$ & 4.20 & $-0.93 *$ & $0.03 *$ & 156.54 & $0.25^{*}$ & $0.67^{\mathrm{ns}}$ \\
\hline G7.45 & 1.31 & $-1.01 *$ & $0.01 *$ & 3.26 & $-0.94 *$ & $0.07 *$ & 23.74 & $-0.88 *$ & $0.14 *$ \\
\hline G7.103 & 2.81 & $-0.96^{*}$ & $0.10^{*}$ & 4.02 & $-0.97 *$ & $0.04 *$ & 75.86 & $-1.78 *$ & $1.59^{\mathrm{ns}}$ \\
\hline G1.62 & 1.54 & $-1.01 *$ & $0.03 *$ & 4.04 & $-1.02 *$ & $0.06^{*}$ & 34.29 & $-0.78 *$ & $0.33 *$ \\
\hline G7.77 & 5.36 & $-1.04 *$ & $0.09 *$ & 3.93 & $-1.03 *$ & $0.04 *$ & 155.81 & $-2.00 *$ & $1.38^{\mathrm{ns}}$ \\
\hline G7.102 & 5.10 & $-1.07 *$ & $0.06^{*}$ & 4.33 & $-0.95^{*}$ & $0.03 *$ & 171.97 & $0.72 *$ & $0.55^{\mathrm{ns}}$ \\
\hline G1.1 & 2.09 & $-0.97 *$ & $0.05^{*}$ & 4.45 & $-1.03 *$ & $0.06^{*}$ & 55.25 & $-2.55^{*}$ & $0.54 *$ \\
\hline G7.32 & 5.03 & $-0.92 *$ & $0.10^{*}$ & 4.31 & $-0.97 *$ & $0.03 *$ & 161.76 & $0.29 *$ & $1.28^{\mathrm{ns}}$ \\
\hline G7.109 & 5.39 & $-1.02 *$ & $0.08^{*}$ & 3.99 & $-0.91 *$ & $0.5^{*}$ & 163.22 & $-0.01 *$ & $1.16^{\mathrm{ns}}$ \\
\hline G7.64 & 3.41 & $-0.93 *$ & $0.10^{*}$ & 3.97 & $0.90 *$ & $0.05 *$ & 90.40 & $-1.65 *$ & $2.55^{\mathrm{ns}}$ \\
\hline G7.85 & 4.63 & $-0.91 *$ & $0.11^{*}$ & 4.18 & $-0.93 *$ & $0.04 *$ & 150.60 & $2.64^{*}$ & $1.78^{\mathrm{ns}}$ \\
\hline G7.25 & 1.31 & $-1.01 *$ & $0.01 *$ & 4.13 & $-0.93 *$ & $0.03 *$ & 24.48 & $-0.86^{*}$ & $0.10 *$ \\
\hline G7.7 & 4.63 & $-1.08 *$ & $0.08 *$ & 4.14 & $-0.93 *$ & $0.05 *$ & 143.06 & $2.36^{*}$ & $1.64^{\mathrm{ns}}$ \\
\hline G7.23 & 5.60 & $-1.02 *$ & $0.08^{*}$ & 4.22 & $-0.94 *$ & $0.03 *$ & 141.82 & $1.98^{*}$ & $1.79^{\mathrm{ns}}$ \\
\hline G7.13 & 4.41 & $-1.04 *$ & $0.08 *$ & 4.09 & $-0.97 *$ & $0.03 *$ & 132.48 & $1.62 *$ & $1.82^{\mathrm{ns}}$ \\
\hline G1.99 & 2.62 & $-1.12 *$ & $0.10^{*}$ & 4.06 & $-1.05^{*}$ & $0.05 *$ & 70.05 & $-0.71 *$ & $1.59^{\mathrm{ns}}$ \\
\hline G1.2 & 5.30 & $-1.10^{*}$ & $0.08^{*}$ & 4.64 & $-0.99 *$ & $0.03 *$ & 185.96 & $-0.94 *$ & $0.80 *$ \\
\hline G7.69 & 1.68 & $-0.96 *$ & $0.03 *$ & 3.84 & $-1.13 *$ & $0.05 *$ & 35.66 & $-0.71 *$ & $0.25 *$ \\
\hline G7.3 & 1.56 & $-1.04 *$ & $0.02 *$ & 4.23 & $-1.06^{*}$ & $0.03 *$ & 34.14 & $-0.95 *$ & $0.25^{*}$ \\
\hline BR3262 & 4.90 & $-0.95 *$ & $0.12 *$ & 3.54 & $-0.94 *$ & $0.04 *$ & 174.91 & $0.11^{*}$ & $1.13^{\mathrm{ns}}$ \\
\hline General mean & 3.10 & & & 3.48 & & & 109.22 & & \\
\hline
\end{tabular}

ns: not significant. * Significant at $5 \%$ probability by $t$-test for $\beta_{1 \mathrm{i}}$ (adaptability) and $\mathrm{F}$ test for $\sigma_{\mathrm{di}}^{2}$ (stability).

For RENA and RESDM, treatments presented low stability and adaptability $\left(\beta_{1 \mathrm{i}}<1\right)$, except for G7.85, with means of 88.97 and $82 \%$, respectively (Table 7); thus, it should have greater than average responses to high BNF potential cultivars (SILVA JÚNIOR et al., 2018).

Based on the methodology of Eberhart \& Russel, the rhizobial isolate G7.85 presented a regression coefficient greater than 1 and higher than that of other rhizobia strains in all variables (adaptability to favorable environments, which we considered to be cultivars with higher BNF efficiency), except for SDM and SNC, which could be indicated for the three cultivars. Strain BR 3262 continued to exhibit better performance and being close to stability should allow consistent BNF dependent on cultivar potential, as shown by Bastos et al. (2012). In contrast, cultivars known to present higher than average BNF potential should be evaluated with $\mathrm{G} 7.85$, if possible, because of its apparent response to higher BNF. 
Table 7. Adaptability and stability estimation of relative efficiency to recommended based on the nitrogen accumulation (RENA) and shoot dry matter (RESDM of rhizobial strains, according to Eberhart e Russel (1966), considering cultivars as environments.

\begin{tabular}{|c|c|c|c|c|c|c|}
\hline \multirow{2}{*}{ Strains } & \multicolumn{3}{|c|}{ RENA } & \multicolumn{3}{|c|}{ RESDM } \\
\hline & Mean & $\beta_{1 \mathrm{i}}$ & $\sigma_{\mathrm{di}}^{2}$ & Mean & $\beta_{1 \mathrm{i}}$ & $\sigma_{\mathrm{di}}^{2}$ \\
\hline G7.14 & 98.18 & $-1.66^{\mathrm{ns}}$ & $0.82 *$ & 105.57 & $-1.16^{*}$ & $0.63^{\mathrm{ns}}$ \\
\hline G7.118 & 98.26 & $-2.27^{\mathrm{ns}}$ & $1.31 *$ & 90.57 & $-1.36^{*}$ & $1.44^{\mathrm{ns}}$ \\
\hline G7.18 & 24.68 & $-0.68^{\mathrm{ns}}$ & $0.55^{\mathrm{ns}}$ & 21.20 & $-0.54 *$ & $0.42^{\mathrm{ns}}$ \\
\hline G7.79 & 15.17 & $-0.85^{*}$ & $0.10^{*}$ & 16.45 & $-0.85^{*}$ & $0.10^{*}$ \\
\hline G7.99 & 89.34 & $-2.61^{*}$ & $1.46^{\mathrm{ns}}$ & 85.67 & $-0.72 *$ & $1.13^{\mathrm{ns}}$ \\
\hline G7.12 & 78.77 & $-0.99^{\mathrm{ns}}$ & $2.59^{\mathrm{ns}}$ & 73.65 & $-0.39 *$ & $2.38^{\mathrm{ns}}$ \\
\hline G7.68 & 92.21 & $-1.30^{\mathrm{ns}}$ & $1.05^{*}$ & 90.16 & $-1.30^{*}$ & $0.77^{\mathrm{ns}}$ \\
\hline G7.45 & 13.69 & $-0.91 *$ & $0.12 *$ & 13.76 & $-0.93 *$ & $0.08^{*}$ \\
\hline G7.103 & 41.25 & $-1.46^{\mathrm{ns}}$ & $1.09^{\mathrm{ns}}$ & 42.53 & $-1.15^{*}$ & $1.19^{\mathrm{ns}}$ \\
\hline G1.62 & 18.98 & $-0.86^{*}$ & $0.26^{\mathrm{ns}}$ & 17.86 & $-0.84^{*}$ & $0.19^{*}$ \\
\hline G7.77 & 92.93 & $-2.91 *$ & $1.46^{\mathrm{ns}}$ & 94.93 & $-0.66^{*}$ & $0.88^{\mathrm{ns}}$ \\
\hline G7.102 & 98.90 & $-0.53^{\text {ns }}$ & $0.62^{\mathrm{ns}}$ & 95.55 & $-0.96^{*}$ & $0.74^{\mathrm{ns}}$ \\
\hline G1.1 & 31.85 & $-2.07 *$ & $0.57 *$ & 30.07 & $-1.76^{*}$ & $0.51^{*}$ \\
\hline G7.32 & 89.12 & $-0.47^{*}$ & $1.00^{\mathrm{ns}}$ & 86.80 & $-0.37 *$ & $1.09^{\text {ns }}$ \\
\hline G7.109 & 95.76 & $-1.15^{\mathrm{ns}}$ & $1.39^{\mathrm{ns}}$ & 97.01 & $-1.45^{*}$ & $0.90^{\text {ns }}$ \\
\hline G7.64 & 52.62 & $-1.13^{*}$ & $2.04^{\mathrm{ns}}$ & 58.39 & $-0.66^{*}$ & $2.35^{\mathrm{ns}}$ \\
\hline G7.85 & 88.97 & $0.35^{*}$ & $2.03^{\mathrm{ns}}$ & 82.00 & $1.15^{*}$ & $1.50^{\mathrm{ns}}$ \\
\hline G7.25 & 14.52 & $-0.89^{\text {ns }}$ & $0.11^{\mathrm{ns}}$ & 13.88 & $-0.91 *$ & $0.09^{*}$ \\
\hline G7.7 & 82.23 & $-0.35^{*}$ & $1.73^{\mathrm{ns}}$ & 81.19 & $-0.99 *$ & $1.40^{\mathrm{ns}}$ \\
\hline G7.23 & 82.01 & $-0.83^{\mathrm{ns}}$ & $1.61^{\mathrm{ns}}$ & 79.42 & $-0.69 *$ & $1.49^{\mathrm{ns}}$ \\
\hline G7.13 & 78.99 & $-1.42^{\mathrm{ns}}$ & $1.79^{\mathrm{ns}}$ & 77.06 & $-0.84 *$ & $1.43^{\mathrm{ns}}$ \\
\hline G1.99 & 46.38 & $-2.03^{*}$ & $2.24^{\mathrm{ns}}$ & 42.20 & $-1.46^{*}$ & $1.69^{\mathrm{ns}}$ \\
\hline G1.2 & 109.23 & $-1.77^{*}$ & $0.89^{\mathrm{ns}}$ & 93.91 & $-1.93 *$ & $0.87^{*}$ \\
\hline G7.69 & 20.92 & $-0.78^{*}$ & $0.28 *$ & 21.05 & $-0.68 *$ & $0.22 *$ \\
\hline G7.3 & 20.31 & $-1.01 *$ & $0.24 *$ & 18.87 & $-1.02 *$ & $0.19^{*}$ \\
\hline BR3262 & 96.39 & $-0.75^{\text {ns }}$ & $0.82 *$ & 96.65 & $-1.25^{*}$ & $0.81^{\mathrm{ns}}$ \\
\hline General mean & 63.44 & & & 61.86 & & \\
\hline
\end{tabular}

ns: not significant. * Significant at $5 \%$ probability by $t$-test for $\beta_{1 \mathrm{i}}$ (adaptability) and $\mathrm{F}$ test for $\sigma_{\mathrm{di}}^{2}$ (stability).

\section{CONCLUSIONS}

The BR 3262 strain proved to be adequate for cowpea seed inoculation with the treatments used, presenting a higher increment than the other rhizobia strains. However, strain G7.85 is of potential interest for higher BNF cultivars.

No compatibility issues were found between the tropical semi-arid soil rhizobial isolates and cowpea cultivars selected for different environments.

The difference in stability of the isolates indicated a potential gain when recommending strains that are better suited for higher potential BNF cultivars.

\section{ACKNOWLEDGEMENTS}

A.C.M.S thanks Fundação de Amparo à Ciência e Tecnologia de Pernambuco (FACEPE, Brazil) IBPG-0134-5.01/12), M. A. L. J. thanks FACEPE (BPV-0008-5.01/19) and CNPq
(304107/2020-4), and F.J.C.F. and T.P.L. thank Coordenação de Aperfeiçoamento de Pessoal de Nível Superior (CAPES, Brazil) for the fellowships provided. All authors express gratitude to CAPES (Finance Code 001), CNPq (401896/2013-7 and 483287/2013-0), and FACEPE (APQ-0453-5.01/15) for financial support.

\section{REFERENCES}

ADU, M. O. et al. Identifying key contributing root system traits to genetic diversity in field-grown cowpea (Vigna unguiculata L. Walp.) genotypes. Field Crops Research, 232: 106-118, 2019.

ALMEIDA, A. L. G. et al. Produtividade do feijãocaupi cv BR 17 Gurguéia inoculado com bactérias diazotróficas simbióticas no Piauí. Revista Brasileira de Ciências Agrárias, 3: 364-369, 2010.

BASTOS, V. J. et al. Avaliação da fixação biológica 
de nitrogênio em feijão-caupi submetido a diferentes manejos da vegetação natural na savana de Roraima. Revista Agroambiente On-line, 6: 133-139, 2012.

BRASIL. Instrução Normativa SDA n ${ }^{\circ} 13$, de 24 de março de 2011. Aprova as normas sobre especificações, garantias, registro, embalagem e rotulagem dos inoculantes destinados à agricultura, bem como as relações dos microorganismos autorizados e recomendados para produção de inoculantes no Brasil, na forma dos Anexos I, II e III, desta Instrução Normativa. Diário Oficial da União, Brasília, DF, 25 mar. 2011. Seção 1, p. 3.

CAMPO, R. J.; HUNGRIA, M. Protocolo para análise da qualidade e da eficiência agronômica de inoculantes, estirpes e outras tecnologias relacionadas ao processo de fixação biológica do nitrogênio em leguminosas. In: Reunião da rede de laboratórios para recomendação, padronização e difusão de tecnologia de inoculantes de interesse agrícola, 13: 89-123, 2006.

CARVALHO, L. R. et al. Nodulation and biological nitrogen fixation (BNF) in forage peanut (Arachis pintoi) cv. Belmonte subjected to grazing regimes. Agriculture, Ecosystems \& Environment, 278: 96106, 2019.

CHAGAS JUNIOR, A. F. et al. Eficiência agronômica de estirpes de rizóbios inoculadas em feijão-caupi no Cerrado, Gurupi-TO. Revista Ciência Agronômica, 41: 709-714, 2010.

EBERHART, S. A.; RUSSELL, W. A. Stability parameters for comparing cultivars. Crop Science, 6: 36-40, 1966.

FAOSTAT, F. FAOSTAT statistical database. Publisher: FAO (Food and Agriculture Organization of the United Nations), Rome, Italy, 2016.

FERREIRA, P. A. A. et al. New rhizobia strains isolated from the Amazon region fix atmospheric nitrogen in symbiosis with cowpea and increase its yield. Bragantia, 78: 38-42, 2019.

GUALTER, R. M. R. et al. Eficiência agronômica de estirpes de rizóbio em feijão caupi cultivado na região da Pré-Amazônia Maranhense. Pesquisa Agropecuária Brasileira, 46: 303-308, 2011.

GONÇALVES, J. R. P. BRS Novaera: cultivar de feijão-caupi para o cultivo em várzeas do Amazonas. Jaguariúna: Embrapa Meio Ambiente, 2012. 4 p. (Comunicado Técnico, 51)
HOAGLAND, D. R.; ARNON, D. I. The waterculture method for growing plants without soil. 2 . ed. Berkeley: California Agricultural Experiment Station, 1950. 32 p.

IBEWIRO, B. et al. Symbiotic performance of herbaceous legumes in tropical cover cropping systems. The Scientific World, 1: 17-21, 2001.

IPA - Instituto Agronômico de Pernambuco. CaupiIPA-206: nova cultivar de feijao macassar (Vigna unguiculata $[\mathrm{L}]$ Walp.) tipo moita para Pernambuco. Recife, PE: Embrapa Amazônia Ocidental, 1989. 2 p.

IRISARRI, P. et al. Selection of competitive and efficient rhizobia strains for white clover. Frontiers in Microbiology, 10: 768, 2019.

JI, J. et al. Genome editing in cowpea Vigna unguiculata using CRISPR-Cas9. International Journal of Molecular Sciences, 20: 2471, 2019.

LEITE, J. et al. Genomic identification and characterization of the elite strains Bradyrhizobium yuanmingense BR 3267 and Bradyrhizobium pachyrhizi BR 3262 recommended for cowpea inoculation in Brazil. Brazilian Journal of Microbiology, 49: 703-713, 2018.

LIRA, M. A. et al. Estabilidade de resposta do capim -braquiária (Brachiaria decumbens, Stapf.) sob níveis crescentes de nitrogênio e fósforo. Pesquisa Agropecuária Brasileira, 29: 1151-1157, 1994.

LIRA, T. P. Diversidade e eficiência simbiótica de isolados rizobianos de solos do semiárido brasileiro. 2014. 45 f. Dissertação (Mestrado em Agronomia: Ciência do Solo) - Universidade Federal Rural de Pernambuco, Recife, 2014.

MALAVOLTA. E. et al. Avaliação do estado nutricional das plantas: Princípios, métodos e técnicas de avaliação do estado nutricional. 1. ed. Piracicaba, SP, 1997. 319 p.

MELO S. R.; ZILLI J. E. Fixação biológica de nitrogênio em cultivares de feijão-caupi recomendadas para o estado de Roraima. Pesquisa Agropecuária Brasileira, 44: 1177-1183, 2009.

NASCIMENTO, L. R. S. et al. Vegetation cover affects rhizobia-tree legume symbiosis in the semiarid region of Brazil. African Journal of Agricultural Research, 14: 770-776, 2019.

NDUNGU, S. M. et al. Cowpea (Vigna unguiculata 
L. Walp) hosts several widespread bradyrhizobial root nodule symbionts across contrasting agroecological production areas in Kenya. Agriculture, Ecosystems \& Environment, 261: 161-171, 2018.

OMOIGUI, L. O. et al. Inheritance of resistance to Cercospora leaf spot disease of cowpea [Vigna unguiculata (L.) Walp]. Euphytica, 215: 101, 2019.

PUOZAA, D. K.; JAISWAL, S. K.; DAKORA, F. D. Phylogeny and distribution of Bradyrhizobium symbionts nodulating cowpea (Vigna unguiculata L. Walp) and their association with the physicochemical properties of acidic African soils. Systematic and Applied Microbiology, 42: 403414, 2019.

SABOYA, R. C. C. et al. Resposta do feijão-caupi a estirpes fixadoras de nitrogênio em Gurupi-TO. Journal of Biotechnology and Biodiversity, 4: 40$48,2013$.

SAKA, J. O. et al. Assessment of varietal diversity and production systems of cowpea (Vigna unguiculata (L.) Walp.) in Southwest Nigeria. Journal of Agriculture and Rural Development in the Tropics and Subtropics, 119: 43-52, 2019.

SANTOS, C. E. R. S. et al. Efetividade de rizóbios isolados de solos da região nordeste do Brasil na fixação do $\mathrm{N}_{2}$ em amendoim (Arachis hypogaea L.). Acta Scientiarum. Agronomy, 27: 301-307, 2005.

SAS INSTITUTE, I. The SAS System for Windows. Cary, North Carolina: SAS Institute Inc, 1999.

SENA, P. T. S. et al. Molecular, physiological, and symbiotic characterization of cowpea rhizobia from soils under different agricultural systems in the semiarid region of brazil. Journal of Soil Science and Plant Nutrition, s/v: 1-15, 2020.

SILVA, A. C. et al. Diagnóstico da produção de feijão-caupi no nordeste brasileiro. Revista da Universidade Vale do Rio Verde, 16: 1-5, 2018.

SILVA JÚNIOR, E. B. et al. Rhizobium inoculation of cowpea in brazilian cerrado increases yields and nitrogen fixation. Agronomy Journal, 110: 722 $727,2018$.

SILVA, F. B. R. et al. Zoneamento Agroecológico do Estado de Pernambuco. Recife, PE: Embrapa Solos - Unidade de Execução de Pesquisa e Desenvolvimento/Governo do Estado de Pernambuco (Secretaria de Produção Rural e
Reforma Agrária), 2001.

SOARES, A. L. L. et al. Eficiência agronômica de rizóbios selecionados e diversidade de populações nativas nodulíferas em Perdões (MG). I - caupi. Revista Brasileira de Ciência do Solo, 30: 795-802, 2006.

STAMFORD, N. P.; NEPTUNE, A. M. L. Especificidade hospedeira e competição entre estirpes de Rhizobium em inoculação cruzada com quatro estirpes de Vigna unguiculata (L.) Walp. Revista Ômega, 3: 25-34, 1979.

VIEIRA, C. L. et al. Inoculação de variedades locais de feijão macassar com estirpes selecionadas de rizóbio. Revista Brasileira de Engenharia Agrícola e Ambiental, 11: 1170-1175, 2010.

VINCENT, J. M. A manual for the practical study of root nodule bacteria. Oxford, Black well Scientific Publications. (IBP Handbook, 15). 1970. $164 \mathrm{p}$. 